\title{
STRUCTURAL DETERMINATION OF L-ASPARAGINASE II OF STREPTOMYCES ALBIDOFLAVUS AND INTERACTION ANALYSIS WITH L-ASPARAGINE AND CEFOTAXIME
}

\section{ACHYUTUNI VENKATA NAGA TEJASWINI, MALOTHU RAMESH*}

\author{
*School of Biotechnology, Institute of Science and Technology, Jawaharlal Nehru Technological University, Kakinada 533003, Andhra \\ Pradesh, India \\ *Email: ramesh_biotech@jntuk.edu.in
}

Received: 05 Mar 2021, Revised and Accepted: 14 May 2021

\begin{abstract}
Objective: L-Asparaginase enzyme possesses a crucial role in the treatment of various hematologic malignancies. The current study focuses on homology modeling and interaction analysis of L-Asparaginase proteins belonging to Streptomyces albidoflavus (S. albidoflavus) with the essential ligand L-Asparagine and subsequent analysis with essential $\beta$-lactam antibiotic Cefotaxime.
\end{abstract}

Methods: The process of understanding Asparaginase interactions primarily involved structure determination of WP_096097608, WP_095730301, which is achieved by GalaxyTBM, I-TASSER and SWISS-MODEL. Further, the S. albidoflavus Asparaginase proteins are subjected to GalaxySite and Autodock Vina of PyRx analysis.

Results: The GalaxyTBM predicted structures of both the proteins are found promising on various validation studies. The two Asparaginase proteins exhibited high binding affinities of-6.8 and-6.5 kcal/mol with Cefotaxime and-5.1 and-4.9 kcal/mol towards Asparagine. The protein WP_096097608 residues forming hydrogen bonds with L-Asparagine are also analysed to involve in interaction with Cefotaxime on individual docking analysis.

Conclusion: The current findings details the two S. albidoflavus Asparaginase proteins affinity towards L-Asparagine, hence can be assessed further for immunogenicity studies. In addition to the above findings, an attempt is made to find the L-Asparaginase binding possibilities with non-metals that identified an essential $\beta$-lactam antibiotic Cefotaxime to be an effective inhibitor. This study helps in understanding the interactions of LAsparaginase with Cefotaxime, as intake of antibiotics between the phases of chemotherapy is observed to treat various infections and also as an antibiotic to microbes that utilize Asparaginase as a vital enzyme.

Keywords: L-Asparaginase, Streptomyces, Homology modelling, GalaxyTBM, I-TASSER, L-Asparagine, Cefotaxime

(C) 2021 The Authors. Published by Innovare Academic Sciences Pvt Ltd. This is an open access article under the CC BY license (https://creativecommons.org/licenses/by/4.0/)

DOI: https://dx.doi.org/10.22159/ijap.2021v13i4.41303. Journal homepage: https://innovareacademics.in/journals/index.php/ijap

\section{INTRODUCTION}

The L-Asparaginase (Asp) enzyme currently administered for Acute Lymphoblastic Leukemia (ALL) is obtained from the Erwinia chrysanthemi and Escherichia coli [1]. The high efficacy of Asp in treating ALL is also observed in diverse groups such as in pubescent and in adults $[2,3]$ apart from children who are highly susceptible to ALL $[4,5]$. Although Asparaginase as a medication possesses a vital role in hematologic malignancy treatment, it is reported that it may aggravate allergic and toxic reactions in some patients [6] which is considered as the prime objective to identify new Asp enzyme sources. As the Asp protein interaction with Asparagine has high significance in the field of medicine in breaking down asparagine into two different compounds aspartic acid and ammonia, thereby initiating the death of the tumor cells.

Antibiotics are highly popular metabolites that are prioritized in treating multiple infections, in particular, display a pivotal role in inhibiting the proliferation of malignant cells and restraining metastasis [7]. Despite the benefits, they are perceived to be detrimental to beneficial microbes [8]. Hence the studies involving essential Asparaginase and antibiotic Cefotaxime can be found useful in understanding the interactions as usage of antibiotics by the cancer patients is observed to treat infections.

The various computational methodologies applied for the current analysis includes template-based assessments and ab initio modeling which are considered prominent to evaluate the protein 3D structure. The computational approach provides a detailed assessment of the properties and interactions of the significant compound thus minimizes the errors and undesirable results during in vitro analysis [9]. The approach towards high accuracy of protein structures involves the execution of energy and structural refinements, retaining similarity to the native conformations $[10$, 11]. A detailed overview of the refined structures and the active sites in quick time is crucial for the formulation of useful therapeutic metabolites [12]. It would be also significant to understand the interaction of Asp enzyme of two S. albidoflavus with Asparagine for quest about alternative enzyme formulations. The current study aims to find the Streptomyces albidoflavus (S. albidoflavus) Asp protein binding potentiality with its natural target inhibitor LAsparagine (Asparagine) and binding possibilities with non-metals.

\section{MATERIALS AND METHODS}

Sequence retrieval and determination of protein characteristics

The Asp protein sequences with accession numbers WP_096097608, WP_095730301 belonging to S. albidoflavus are obtained in FASTA format from the National Center for Biotechnology Information database [13]. The protein sequences are searched for similar entries with the application of BLAST tool [14] in the UniProtKB database, a prioritized protein knowledgebase to maintain accurate protein information with access to entries from different protein data sources [15]. The sequence alignment of the query protein sequences and the resulted protein sequences are analyzed by ClustalW [16] and individual phylogenetic trees are built by the Neighbor-joining method [17] with the default criteria of 1000 bootstrap values [18] in the desktop version of the MEGA X application [19]. The proteins are assessed by the ExPASy ProtParam studies to evaluate the physicochemical features, similarities and differences in the characteristics of two Asp proteins [20].

Asp proteins structural elucidation and molecular annotation

The Asp proteins are evaluated for the presence of helix, Pi helix, strands, turns, coils and beta bridges by the SOPMA programme [21]. In addition, brief insights into the secondary structure are also provided by the I-TASSER along with the confidence score [22]. Besides, the proteins are further subjected to analysis with NCBICDD [23], Pfam database [24]. Structural evaluation of Asp proteins 
is achieved by SWISS-MODEL [25], GalaxyTBM [26] and I-TASSER [22]. Refinement of the resulted models is performed by Galaxy Refine [27] followed by assessment with PROCHECK [28] ProSA web server and ERRAT analysis [29, 30].

\section{Molecular docking studies of Asp proteins}

The binding pockets, functional annotations of the proposed protein models are fetched by two docking methodologies namely GalaxySite [31] and AutoDock Vina 1.1.2 [32], an integrated methodology of PyRx 0.8 [33]. GalaxySite program is executed by embedding structure determination methods namely GalaxyTBM [26] template identification by HHsearch and protein-ligand docking approach by LigDockCSA [34, 35]. The docked results of Asp proteins are finally visualized in Discovery studio v21 [36].

\section{RESULTS AND DISCUSSION}

The Asp protein sequences of $S$. albidoflavus featuring accession numbers WP_096097608, WP_095730301 are selected for the current study. The sequences are described as non-redundant as per the literature available [13].

\section{Phylogenetic analysis of Asp proteins}

The Asp protein sequences are analyzed for similar proteins by the application of BLAST tool [14] in the vast UniProtKB database [15] and the resulted sequences are aligned by the ClustalW [16] the phylogenetic relationship is derived by the Neighbor-joining method [17] of the MEGA X version [19]. The protein WP_096097608 exhibited clade formation with Asparaginase proteins of the same species with accession number A0A2A2UM91 (99.7\% sequence similarity) and with Streptomyces sp. FR-008 (A0A0S1UPU4) ( $99.2 \%$ sequence similarity) (fig 1A). Similarly, the protein with accession number A0A2A2UM91 of S. albidoflavus appeared $100 \%$ identical categorizing into the same clade with WP_095730301 (fig 1B). In addition, many Asparaginase protein sequences belonging to unspecified species of the Streptomyces family appeared to be in clusters with the query proteins as represented in fig $1 \mathrm{~A}$ and $1 \mathrm{~B}$. A protein of $S$. koyangensis A0A385DBJ8 is observed to be $95.9 \%$ and 96.1\% identical with the two Asp proteins WP_096097608 and WP_095730301 accordingly indicating a phylogenetic relationship. Thus the analysis enables interpretation of the protein characteristics depending on the level of sequence identity. Fig.1A and $1 \mathrm{~B}$ details the phylogenetic tree of the Asp protein sequences that exhibited identity greater than $95 \%$ with different entries of UniProtKB [15].

\section{Analysis of Asp protein sequence characteristics}

The physicochemical profiling of the proteins by the ExPASY ProtParam tool are detailed in table 1 . The protein sequences WP_096097608 is found to possess 328 amino acids (AA) corresponding to 4808 atoms while WP_095730301 possessed 309 AA with 4555 atoms. The molecular weight of the sequences WP_096097608, WP_095730301 are found to be $34171.28 \mathrm{Da}$, 32318.17 Da respectively. The instability index findings categorized the proteins to be stable with an index value found within the acceptable range. The proteins Aliphatic index (AI) ranged 90.34, 92.72 as described in table 1 , the AI values observed between 40-77 and values $>100$ are also reported by Manochitra and team, and also by Appaiah and colleagues [37, 38]. The GRAVY values of $S$. albidoflavus Asp proteins are determined to be 0.058 and 0.090, identifying the proteins as hydrophobic. Similar positive values of 0.106, 0.237 are reported by Bagag and his team [39]. Methionine is identified as an N-terminal for the two proteins is considered significant in measuring the half-life of the protein [20]. The half-life assessments are sum up between 10 to $30 \mathrm{~h}$ as per the studies pertaining to E. coli, yeast and mammalian reticulocytes.

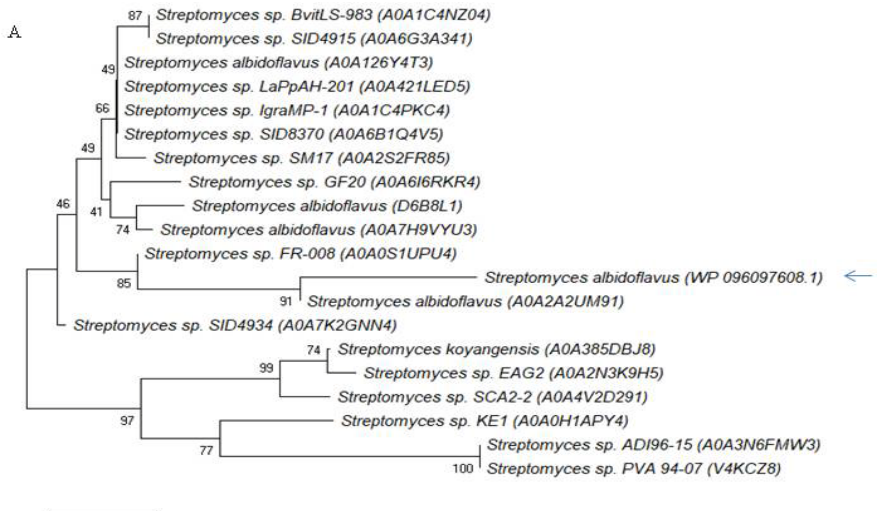

0010

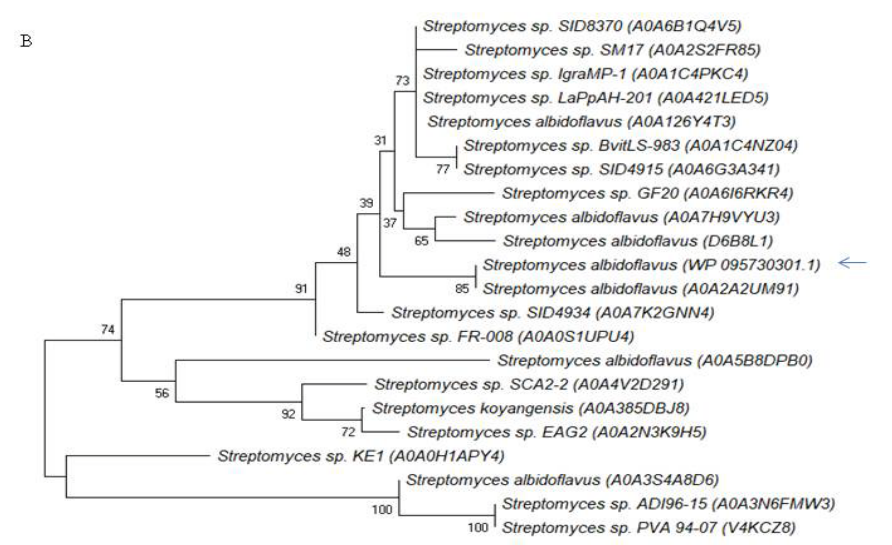

Fig. 1: Phylogenetic tree of $[A]$ WP_096097608, [B] WP_095730301 with protein sequences of UniProtKB constructed by Neighbor-joining method of MEGA X version 
Table 1: ProtParam assessment of the $S$. albidoflavus Asp protein sequences. AA count: Amino acid count; Mwt: Molecular weight; pI value: Theoretical pI; (-)r: negatively charged residues; (+)r: positively charged residues; Ec: Extinction coefficient; CC: Cys residues form cystines; CR: Cys residues are reduced; HL: Half-life; I Index: Instability index; A Index: Aliphatic Index

\begin{tabular}{|c|c|c|c|}
\hline \multirow[t]{2}{*}{ Parameters } & & \multicolumn{2}{|l|}{ Sequence details } \\
\hline & & WP_096097608 & WP_095730301 \\
\hline AA count & & 328 & 309 \\
\hline MWt (Da) & & 34171.28 & 32318.17 \\
\hline pI value & & 6.11 & 5.75 \\
\hline$(-)$ residues & & 36 & 35 \\
\hline$(+)$ residues & & 33 & 30 \\
\hline Formula & & $\mathrm{C}_{1496} \mathrm{H}_{2412} \mathrm{~N}_{444} \mathrm{O}_{440} \mathrm{~S}_{16}$ & $\mathrm{C}_{1422} \mathrm{H}_{2287} \mathrm{~N}_{415} \mathrm{O}_{417} \mathrm{~S}_{14}$ \\
\hline \multirow[t]{2}{*}{ Ec value } & $\mathrm{CC}$ & 22835 & 22710 \\
\hline & $\mathrm{CR}$ & 22460 & 22460 \\
\hline \multirow[t]{2}{*}{ HL (hr) } & in vitro & 30 & 30 \\
\hline & invivo & 20,10 & 20,10 \\
\hline II value & & 35.52 & 33.68 \\
\hline AI value & & 90.34 & 92.72 \\
\hline Gravy & & 0.058 & 0.09 \\
\hline
\end{tabular}

\section{Asp proteins structural elucidation and molecular annotation}

The secondary structure evaluation of Asp proteins by SOPMA exhibited varied results with high dominance of random coils (Cc) in WP_096097608 with $38.72 \%$, followed by $34.76 \%$ of alpha-helices (Hh), $15.85 \%$ of the extended strand (Ee) and $10.67 \%$ of beta turns(Tt). The protein WP_095730301 is determined to be rich in alpha-helical formation with $40.45 \%$ of $\mathrm{Hh}, 33.66 \%$ of Cc, $14.89 \%$ of Ee, $11 \%$ of $\mathrm{Tt}$. The secondary structure possessing alpha-helices (three long and four short lengths), four beta-strands and a set of coils and strands laid between the helices is described by PSIPRED during the I-TASSER WP_096097608 structural evaluation process [40]. Similarly, WP_095730301 is described to be made of four betastrands and six (four long and two short lengths) alpha-helices which are interlinked with each other by a short length coil. The functional annotations of WP_096097608, WP_095730301 on prediction with NCBI-CDD and Pfam servers identified the proteins to be members of PF06089, L-Asparaginase II protein family with alignments between 22 325 AA, 3 306 AA. The NCBI-CDD results are also in agreement with Pfam analysis as the proteins belong to pfam06089-L-Asparaginase II superfamily. The residue range 24326, 5-307 has an E-value 1.18e-137 and 7.13e-138 respectively.

The application of I-TASSER and GalaxyTBM resulted in five 3D protein models by each tool while the SWISS-MODEL resulted in numerous predictions displaying identity between the target and the templates. The target-template identity analysed by SWISS-MODEL is found to be low for both the proteins of interest. The predictions of GalaxyTBM and I-TASSER are considered for further structure validation and active site prediction methods.
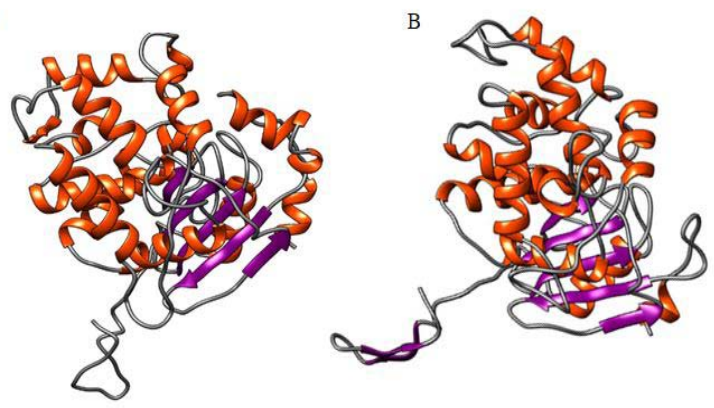

Fig. 2: GalaxyTBM predicted structures of [A] WP_096097608 [B] WP_095730301

GalaxyTBM incorporates multiple templates for structure determination, supported with ab initio modeling of loops to enhance the quality of the predicted models [26]. The GalaxyTBM prediction of WP_096097608 (fig. 2A) is coupled with refinement between the residues 109-117 while unreliable region ranging between 90-98, 120-128, 167-181 are refined for WP_095730301 (fig. 2B). The templates considered for the structural development of the target are found common inferring similarity between the predicted structures of two proteins. The templates 5W2J_B, 407D_A, 1MKI_B, 3IHB_A are evaluated for WP_096097608,407D_A, 1MKI_B, 5W2J_B, 4BQM_B, 3IHB_A for WP_095730301.

The I-TASSER predictions of $S$. albidoflavus Asp protein sequences resulted in protein models scored individually between-5 to 2 , termed as Confident score (C-score) [22]. The first predicted model of WP_096097608 is observed to have a TM score of $0.36 \pm 0.12$ and RMSD $14.0 \pm 3.9 \AA$, C-score 3.13. The models between 2 to 5 possessed a C-score of-3.95,-4.04,-4.16,-4.27. The predicted protein models of WP_095730301 exhibited a C-score of-2.83,-4.01,-3.61,-5 and-3.43. The first model with a C-score of- 2.83 is estimated to have a TM-score of $0.39 \pm 0.13$, RMSD $13.0 \pm 4.2 \AA$. The PDB hits $1 \mathrm{kykA}$, 1ci9A, 3h2gA, 6vm5A, 1k474, 1xkz, $4 \mathrm{mnr}$ are the top aligned templates with normalized Z-score values greater than 1 , considered as best alignment for WP_096097608. The TM-score and C-score of every protein model are considered crucial to understanding the protein characteristics. The TM-scores of the PDB entries with close structural identity exhibited a TM-score between $0.669-0.688$, the highest TM-score of 0.688 is exhibited between the target protein and 3 dwkC. The top-aligned PDB structures structurally similar PDB hits related to WP_095730301 are found identical to WP_096097608 protein structure prediction. However, the TMscore of the structurally close proteins to WP_095730301 displayed much higher values ranging between $0.655-0.701$, 2 wafA being the most structurally similar protein.

The structural refinement is achieved by GalaxyRefine followed by assessment by PROCHECK for the finest models predicted by GalaxyTBM and I-TASSER are described in table 2. The prominent model of WP_096097608 predicted by GalaxyTBM with 87.1 \% (230 AA), $9.8 \%$ (26 AA), 2.3\% (6 AA) of residues in the Ramachandran plot [41] is detailed in fig. 3A while WP_095730301 displayed 89.3 $\%$ (225 AA), $8.3 \%$ (21 AA), $0.8 \%$ (2 AA) of residues (fig. 3B) in the favoured, additionally and generously allowed regions sequentially, representing an acceptable quality proteins. The finest predicted model of I-TASSER exhibited a quality enhanced structure after refinement with $79.2 \%, 16.3 \%, 0.8 \%$ of AA residues referring to WP_096097608, 84.9\%,11.1\%,1.2\% by WP_095730301 in the favoured, additionally and generously allowed segment. The proteins had persistent AA in the disallowed region with 3.8\% (10 AA) and $2.8 \%(7 \mathrm{AA})$ referring to the proteins sequentially. The impact of the AA on the stability and stereochemistry of the protein from the disallowed region is comparatively less in the GalaxyTBM protein models with $0.8 \%(2 \mathrm{AA}), 1.6 \%$ (4 AA) than in the I-TASSER predicted models. 
Table 2: Validation of Asp protein models determined by I-TASSER and GalaxyTBM, Asp protein 1: WP_096097608; Asp protein 2: WP_095730301

\begin{tabular}{|c|c|c|c|c|c|}
\hline \multirow[t]{2}{*}{ Validation tool } & \multirow[t]{2}{*}{ Regions } & \multicolumn{2}{|c|}{ Asp protein 1} & \multicolumn{2}{|c|}{ Asp protein 2} \\
\hline & & GalaxyTBM & I-TASSER & GalaxyTBM & I-TASSER \\
\hline \multirow[t]{4}{*}{ PROCHECK } & Favoured & 87.1 & 79.2 & 89.3 & 84.9 \\
\hline & additionally allowed & 9.8 & 16.3 & 8.3 & 11.1 \\
\hline & generously allowed & 2.3 & 0.8 & 0.8 & 1.2 \\
\hline & Disallowed & 0.8 & 3.8 & 1.6 & 2.8 \\
\hline ProSA web & Z-score & -5.31 & -7.64 & -5.3 & -6.26 \\
\hline ERRAT & & 86.039 & 79.365 & 89.161 & 80.208 \\
\hline
\end{tabular}
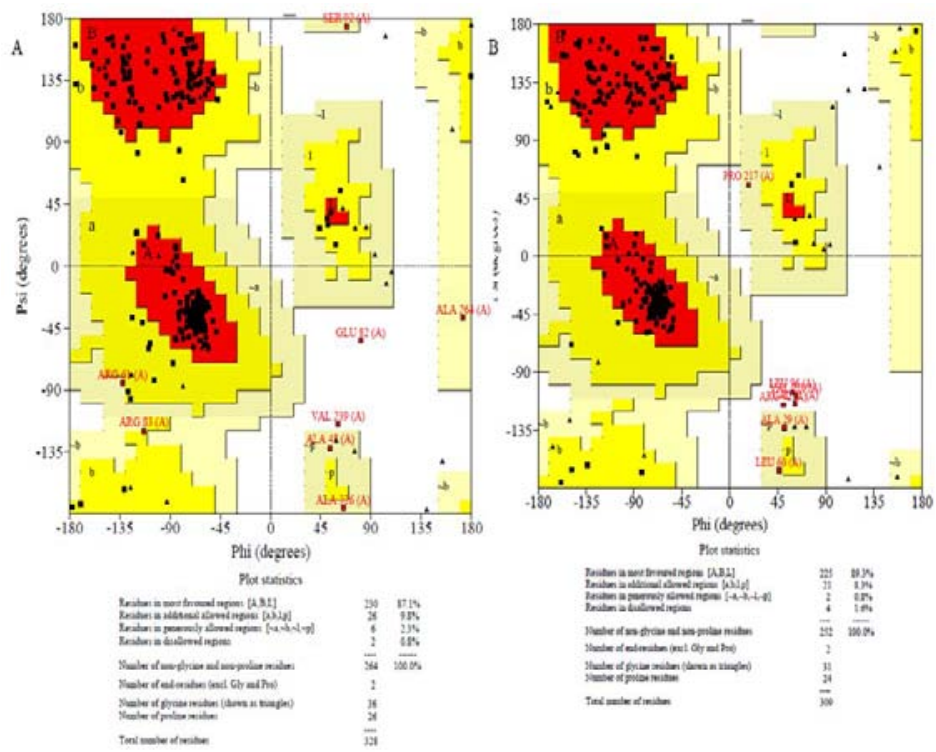

Fig. 3: Ramachandran plots of S. albidoflavus proteins determined by GalaxyTBM analyzed by PROCHECK application. [A] WP_096097608 [B] WP_095730301
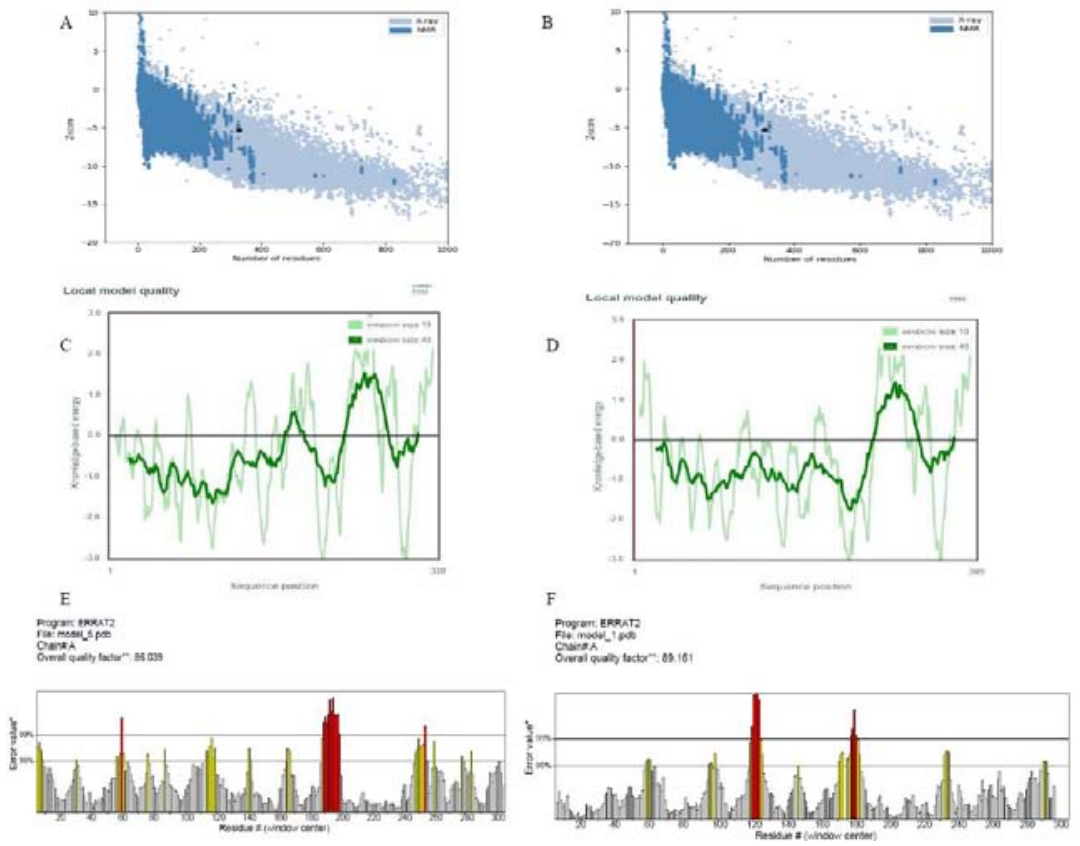

Fig. 4: Validation of S. albidoflavus Asp proteins determined by GalaxyTBM. Panel A, B details the ProSA analysis of WP_096097608 and WP_095730301 and panel C, D details the energy plot of the proteins respectively. The ERRAT quality analysis of WP_096097608 and WP_095730301 is represented by the panel's E and F accordingly

Fig. 4A and 4B depicts the "degree of nativeness" of proteins termed as Z-score resulted in in-5.31,-5.3 by ProSA web on analysis of the protein models determined from WP_096097608 and WP_095730301 sequences accordingly. The assessment of the 
individual model quality by the residual energy plot detailed the models to have a majority of negative residual interaction with peaks generated below the baseline. Minimal exceptional positive peaks have emerged with a slight rise in positive energy concerning the model obtained from WP_096097608 (fig. 4C). Regarding the model obtained from WP 095730301, a substantial decrease in positive energy is observed with only a single peak formation (fig. 4D). However, the total residual energies of both the models remained negative thus they are considered acceptable with scores in the approved range of other pre-determined proteins. Fig. 4E and $4 \mathrm{~F}$ illustrate favourable ERRAT scores of 86.039, 89.161 indicating high-quality proteins as an ERRAT score above 50 falls into an acceptable range [30].

The structure elucidated by GalaxyTBM is found promising, stable, with good stereochemistry among the protein models predicted by ITASSER and SWISS-MODEL as the greater than $90 \%$ of residues are resulted favuorable [28]. The resulted ProSA and ERRAT values are also found significant in comparison to the validation results of ITASSER predicted models. Therefore the GalaxyTBM predicted structure is chosen for further analysis on protein-ligand interaction with a therapeutic application.

\section{Analysis of Asp protein by Galaxysite tool}

As both the proteins belong to Asparaginase II, coincidentally all the templates for the ligand prediction are found to be structures of Penicillin-binding proteins as predicted by the GalaxySite tool however, the source organisms differed. The protein WP_096097608 is identified to bind to Ligand CEF (CEFOTAXIME) and CDS. Similarly, the Asp protein WP_095730301 displayed interactions with Ligands CDS and BMG. The interactions of the two proteins with the respective ligand are displayed through LIGPLOT [42] (fig. 5).

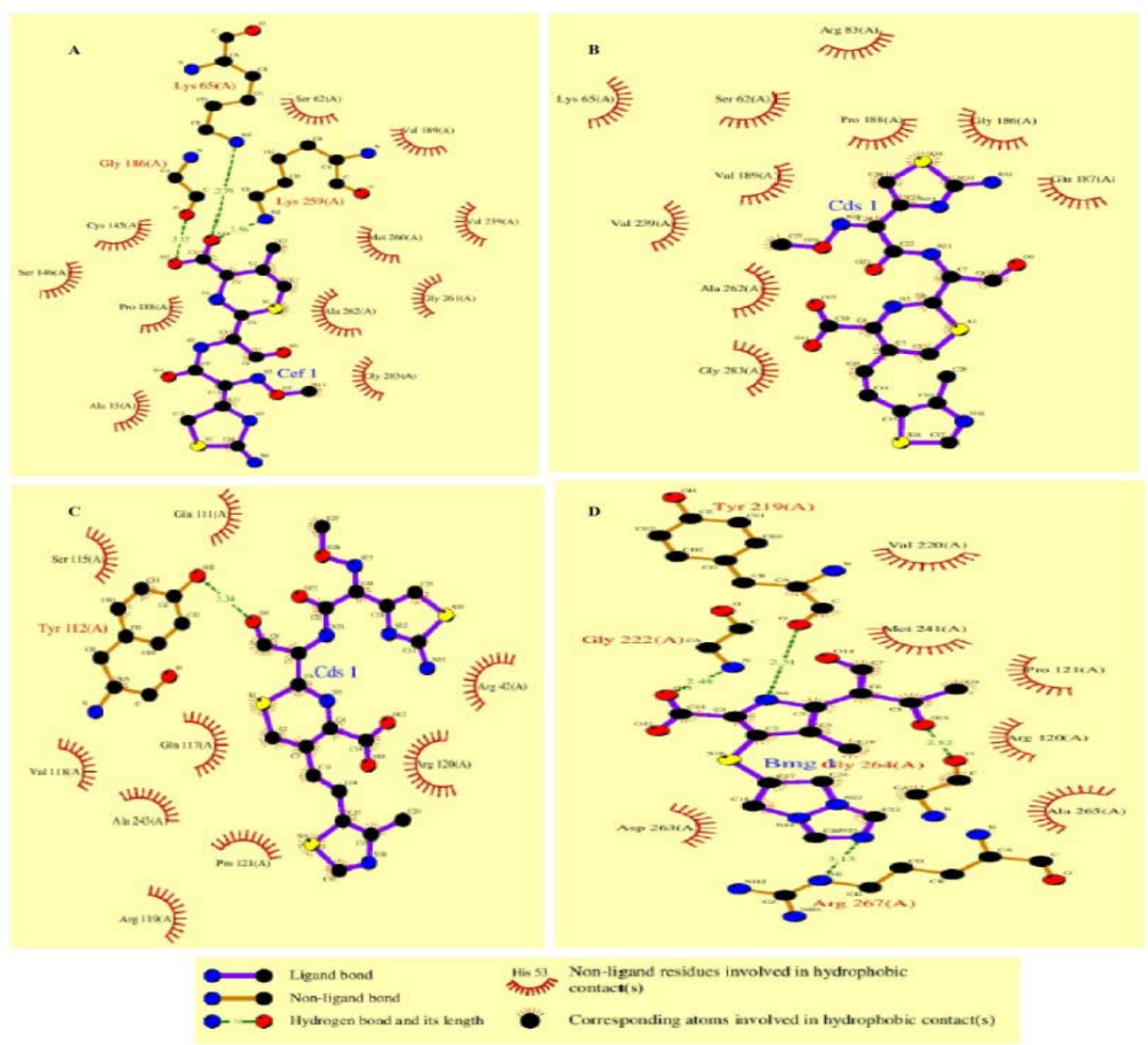

Fig. 5: GalaxySite predicted interactions of Asp proteins visualized by Ligplot: [A] WP_096097608 with Cef (Cefotaxime) [B] WP_096097608 with Cds [C] WP_095730301 with Cds [4] WP_095730301 with Bmg

CEF binds to WP_096097608 chain A at Gly 14, Ala 15, Gly 16, Pro 17, Ser 62, Lys 65, Cys 145, Ser 146, Gly 186, Glu 187, Pro 188, Val 189, Val 239, Lys 259, Met 260, Gly 261, Ala 262 and Gly 283. Hydrogen bonds formation is observed between 01 atom of Cefotaxime and Lysine 65, 259 residues of protein WP_096097608. Hydrophobic interactions are also observed between Cefotaxime and Ala 15, Ser 62, Cys 145, Ser 146, Pro 188, Val 189, Val 239, Met 260, Gly 261, Ala 262, Gly 283 of WP_096097608.

The ligand CDS interacts with 16 AA residues of WP_096097608, namely ser Ser 62S, Lys 65, Arg 83R, Cys 145, Ser 146, His 149, Gly 186, Glu 187, Pro 188, Val 189, Val 239, Lys 259, Met 260, Ala 262, Gly 283 and Ala 284. CDS also bind with amino acid residues of WP_095730301 at ARG 42, GLU 108, GLN 111, TYR 112, SER 115, GLN 117, VAL 118, ARG 119, ARG 120, Pro 121, Ala 243, GLU 244. WP_095730301 is identified to bind with BMG at ARG 120,PRO
121,TYR 219, VAL 220, ALA 221, GLY 222, MET241, GLY 242, Asp 263, GLY 264, ALA 265, ARG 267.

Among the above-identified non-metals compatible for binding with Asp proteins, Cef is known to be a $\beta$-lactam antibiotic with application in treating pneumonia [43] and also effective when provided to cancer patients to treat urinary tract infection, septicemia as per Rolston and team [44]. The antibiotic Cefotaxime in combination with Ciprofloxacin and Ofloxacin is reportedly found to be used in the treatment of neutropenia among cancer patients $[45,46]$. The studies revealed that Cefotaxime is prescribed to all age groups including neonates [47].

Although GalaxySite analysis identified Cefotaxime binding to Asp protein WP_096097608, both the proteins binding residues and possibilities with Cefotaxime is studied in addition to Asparagine by 
individual docking based on the interpretation that two Asp proteins belong to pfam06089 L-Asparaginase II superfamily.

\section{Molecular docking analysis of Asp proteins by Pyrx application}

The docking methodology of the Asp proteins is executed by Galaxysite and by Pyrx application. The ligands Asparagine (identifier 6267) and Cefotaxime (identifier 5742673) obtained from Pubchem [48] are subjected to blind docking by PyRx 0.8 application comprising an integrated AutoDock Vina version 1.1.2 tool. The Openbabel [49], an inbuilt version of Pyrx tool with a united force field and Chimera 1.14 [50], a desktop application with default criteria of minimization are utilized to minimize and convert Asparagine and Cefotaxime into pdbqt format respectively. The predicted best structures of the proteins WP_096097608 and WP_095730301 are considered as macromolecules for analysis with the ligands sequentially. Nine predicted binding poses are identified for each individual AspAsparagine, Asp-Cefotaxime combinations. The Asp protein WP_096097608 displayed a binding affinity of-5.1 kcal/mol,-6.8 $\mathrm{kcal} / \mathrm{mol}$ towards Asparagine and Cefotaxime respectively, followed by-4.9 kcal $/ \mathrm{mol},-6.5 \mathrm{kcal} / \mathrm{mol}$ for Asparagine and Cefotaxime accordingly by WP_095730301. The interpretation confirms both the ligands possess strong interactions towards WP_096097608.

The Asp protein WP_096097608 exhibited three types of bonds with Asparagine (fig. 6A) comprising LYS 65, CYS 145, LYS 259, ALA 262 with Hydrogen bond while SER 62 appeared with hydrogen and carbon-hydrogen bond. ARG 83, GLY 186, GLU 187, PRO 188, VAL 189, MET 260, GLY 261, GLY 283 are found surrounding the ligand exhibiting van der Waal interactions.

The Asp protein WP_096097608, Cefotaxime docked framework (fig. $6 \mathrm{~B}$ ) is observed to have six types of bonds consisting of $\mathrm{H}$ bonds with
GLY 14, SER 62, LYS 65, LYS 259. Gly 186 forming H bond with OH group of the ligand while ASP282 displayed an attractive charge and a $\pi$-anion. CYS 145, VAL 239 displayed with alkyl bonds in addition, the ligand is associated with thirteen residues featuring van der Waals interactions, namely ASP 13, ALA 15, PRO 17, ARG 83, SER 146, GLU187, PRO 188, VAL 189, GLY 261, ALA 262, GLY 283, ALA 284, ARG 286 and an additional GLY 161 with an unfavourable donor-donor bond. The Asp protein WP_096097608 and Cefotaxime docking analysis is in coincidence with the Galaxysite interpretation. The template 3VSL_B, structure of Penicillin-binding protein with methicillin resistance is identified by the GalaxySite analysis in the identification of ligand Cefotaxime concerning WP_096097608. Similar molecular docking analysis with binding energy-4.66 $\mathrm{kcal} / \mathrm{mol}$ involving Asp enzyme and Ciproflaxin is earlier reported by Vimal and team [51].

The protein WP_095730301 docked with Asparagine (fig. 6C) exhibited Conventional H bonds with only ASN 45 and THR 161 and van der Waals forces with ARG 42, SER 43, LYS 46, GLN 49, GLU 63, ALA 66, LEU 67, TYR 112, ALA 164, ALA165 residues. The Cefotaxime and WP_095730301 docked pattern (fig. 6D) is visualised to have salt bridges and $\pi-\pi$ stacked bonds in addition to the bonds displayed by WP_096097608 however, no unfavorable bonds are observed. GLY 57, TYR 147 showed a carbon-hydrogen bond while ASP 59 appeared to be a Hydrogen bond with an oxygen atom. Multiple bonds are observed by HIS 72 with a $\pi-\pi$ stacking and two $\mathrm{H}$ bonds anticipated for the overall stability of the AspCefotaxime protein. Similarly, ASP 149 also possesses multiple interactions with a carbon-hydrogen bond, salt bridge, $\pi$-anion and hydrogen bond. In addition, ILE 53, LEU residues 58, 65, 144 and 148, ALA residues 68 and 69, SER 71 and 146, ASP 145 exhibited van der Waal bonds.
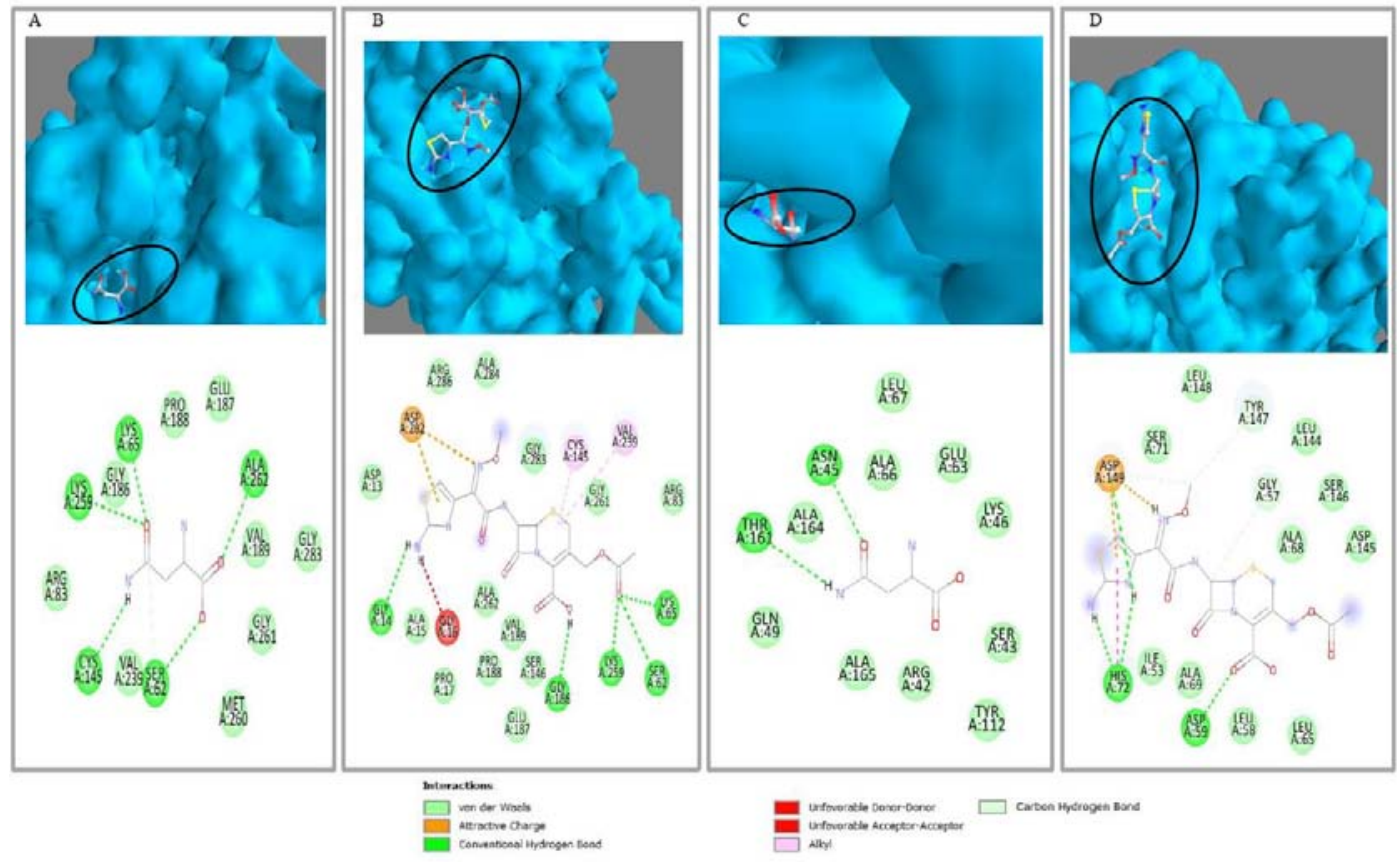

Fig. 6: Molecular Docking studies: [A] WP_096097608 with Asparagine [B] WP_096097608 Cefotaxime [C] WP_095730301 with Asparagine [D] WP_095730301 with Cefotaxime. The upper half of each panel represents 3d surface images obtained from PyRx analysis while the lower part of each panel displays 2D interactions illustrated by the Discovery studio

It is observed that the GalaxyTBM predicted Asp proteins structures on subjecting to docking studies, reported favourable results. Specifically, Asp-Asparagine docked conformation with-5.1 and-4.9 $\mathrm{kcal} / \mathrm{mol}$ is within the acceptable binding affinity interpretations by different researchers studied in different organisms such as in Salmonella typhimurium, Pectobacterium carotovorum, Streptomyces scabrisporus reported binding energy of-5.49,-5.51,-4.62 kcal/mol respectively concerning the docking analysis with Asparagine [51-
53]. Although much higher binding affinities are expressed in different sources of Asp enzyme, the current analysis focuses on new insights of Asp-Asparagine confirmation from two S. albidoflavus proteins.

The Autodock Vina predictions concerning Asp protein WP_096097608 exhibiting an affinity of-6.8 kcal/mol towards Cefotaxime despite the presence of an unfavorable bond which is 
considered to impact the stability of the protein. On contrary, no such unfavourable bonds appeared with WP_095730301 docked structure exhibiting binding energy- $6.5 \mathrm{kcal} / \mathrm{mol}$ is also considered to enhance the stability due to the hydrogen and stacked $\pi$ bonds.

\section{CONCLUSION}

The present study on $S$. albidoflavus Asparaginase sequences identified the protein structures with stable 86.039, 89.161 ERRAT scores. The Asp protein WP_096097608 is found dominant with binding energy higher than WP_095730301 protein towards Asparagine. Thus the Asp homology and Asp-Asparagine docking analysis are considered as a successful step towards the quest for alternative Asp sources which may further be evaluated for molecular dynamic simulations and immunogenicity assessments. Similarly, the Asp-Cefotaxime interaction may be beneficial during anti-tumor drug development and also may be further analysed as an effective antibiotic to microbes that require Asp as a necessary enzyme.

\section{ACKNOWLEDGEMENT}

The authors extend their sincere gratitude to the School of Biotechnology, JNTUK by providing various instrumental facilities.

\section{FUNDING}

There are no funds received for the current research work.

\section{AUTHORS CONTRIBUTIONS}

Both the authors have equally contributed to the current study.

\section{CONFLICT OF INTERESTS}

The authors declare no conflicts of interest.

\section{REFERENCES}

1. Ehsanipour EA, Sheng X, Behan JW, Wang X, Butturini A, Avramis $\mathrm{VI}$, et al. Adipocytes cause leukemia cell resistance to Lasparaginase via release of glutamine. Cancer Res 2013;73:2998-6.

2. Barry E, DeAngelo DJ, Neuberg D, Stevenson K, Loh ML, Asselin $\mathrm{BL}$, et al. Favorable outcome for adolescents with acute lymphoblastic leukemia treated on dana-farber cancer institute acute lymphoblastic leukemia consortium protocols. J Clin Oncol 2007;25:813-9.

3. Rowe JM, Buck G, Burnett AK, Chopra R, Wiernik PH, Richards $\mathrm{SM}$, et al. Induction therapy for adults with acute lymphoblastic leukemia: results of more than 1500 patients from the international ALL trial: MRC UKALL XII/ECOG E2993. Blood 2005;106:3760-7.

4. Moghrabi A, Levy DE, Asselin B, Barr R, Clavell L, Hurwitz C, et al. Results of the dana-farber cancer institute all consortium protocol 95-01 for children with acute lymphoblastic leukemia. Blood 2007;109:896-904.

5. Katz AJ, Chia VM, Schoonen WM, Kelsh MA. Acute lymphoblastic leukemia: an assessment of international incidence, survival, and disease burden. Cancer Causes Control 2015;26:1627-42.

6. Mitchell L, Hoogendoorn H, Giles AR, Vegh P, Andrew M. Increased endogenous thrombin generation in children with acute lymphoblastic leukemia: risk of thrombotic complications in L'Asparaginase-induced antithrombin III deficiency. Blood 1994;83:386-91.

7. Gao Y, Shang Q, Li W, Guo W, Stojadinovic A, Mannion C, et al. Antibiotics for cancer treatment: a double-edged sword. J Cancer 2020;11:5135-49.

8. Reuter G. The lactobacillus and bifidobacterium microflora of the human intestine: composition and succession. Curr Issues Intest Microbiol 2001;2:43-53.

9. Balakrishnan NA, Raj JS, Kandakatla NA. In silico studies on new indazole derivatives as gsk-3 $\beta$ inhibitors. Int J Pharm Pharm Sci 2015;7:295-9.

10. Feig M. Computational protein structure refinement: almost there, yet still so far to go. Wiley Interdiscip Rev Comput Mol Sci 2017;7:1307.

11. Shuid AN, Kempster R, Mcguffin LJ. ReFOLD: a server for the refinement of 3D protein models guided by accurate quality estimates. Nucleic Acids Res 2017;45:422-8.
12. Kinoshita K, Nakamura H. Protein informatics towards function identification. Curr Opin Struct Biol 2003;13:396-400.

13. Agarwala R, Barrett T, Beck J, Benson DA, Bollin C, Bolton E, et al. Database resources of the national center for biotechnology information. Nucleic Acids Res 2018;46:8-13.

14. Altschul SF, Gish W, Miller W, Myers EW, Lipman DJ. Basic local alignment search tool. J Mol Biol 1990;215:403-10.

15. UniProt Consortium. The universal protein resource (UniProt). Nucleic Acids Res 2007;36:D190-5.

16. Thompson JD, Higgins DG, Gibson TJ. CLUSTAL W: improving the sensitivity of progressive multiple sequence alignment through sequence weighting, position-specific gap penalties and weight matrix choice. Nucleic Acids Res 1994;22:4673-80.

17. Saitou N, Nei M. The neighbor-joining method: a new method for reconstructing phylogenetic trees. Mol Bio Evol 1987;4:406-25.

18. Felsenstein J. Confidence limits on phylogenies: an approach using the bootstrap. Evolution 1985;39:783-91.

19. Kumar S, Stecher G, Li M, Knyaz C, Tamura K. MEGA X: molecular evolutionary genetics analysis across computing platforms. Mol Biol Evol 2018;35:1547-9.

20. Gasteiger E, Gattiker A, Hoogland C, Ivanyi I, Appel RD, Bairoch A. ExPASy: the proteomics server for in-depth protein knowledge and analysis. Nucleic Acids Res 2003;31:3784-8.

21. Geourjon C, Deleage G. SOPMA: significant improvements in protein secondary structure prediction by consensus prediction from multiple alignments. Bioinformatics 1995;11:681-4.

22. Yang J, Zhang Y. I-TASSER server: new development for protein structure and function predictions. Nucleic Acids Res 2015;43:174-81.

23. Lu S, Wang J, Chitsaz F, Derbyshire MK, Geer RC, Gonzales NR, et al. CDD/SPARCLE: the conserved domain database in 2020. Nucleic Acids Res 2020;48:265-8.

24. Mistry J, Chuguransky S, Williams L, Qureshi M, Salazar GA, Sonnhammer ELL, et al. Pfam: the protein families database in 2021. Nucleic Acids Res 2021;49:412-9.

25. Waterhouse A, Bertoni M, Bienert S, Studer G, Tauriello G, Gumienny $\mathrm{R}$, et al. SWISS-MODEL: homology modelling of protein structures and complexes. Nucleic Acids Res 2018;46:296-303.

26. Ko J, Park H, Seok C. GalaxyTBM: template-based modeling by building a reliable core and refining unreliable local regions. BMC Bioinformatics 2012;13:1-8

27. Heo L, Park H, Seok C. GalaxyRefine: protein structure refinement driven by side-chain repacking. Nucleic Acids Res 2013;41:384-8

28. Laskowski RA, MacArthur MW, Moss DS, Thornton JM. PROCHECK: a program to check the stereochemical quality of protein structures. J Appl Crystallogr 1993;26:283-91.

29. Wiederstein M, Sippl MJ. ProSA-web: interactive web service for the recognition of errors in three-dimensional structures of proteins. Nucleic Acids Res 2007;35(Suppl 2):407-10.

30. Colovos C, Yeates TO. Verification of protein structures: patterns of nonbonded atomic interactions. Protein Sci 1993;2:1511-9.

31. Heo L, Shin WH, Lee MS, Seok C. GalaxySite: ligand-binding-site prediction by using molecular docking. Nucleic Acids Res 2014;42:210-4.

32. Trott 0 , Olson AJ. AutoDock vina: improving the speed and accuracy of docking with a new scoring function, efficient optimization, and multithreading. J Comput Chem 2010;31:455-61.

33. Dallakyan S, Olson AJ. Small-molecule library screening by docking with PyRx. Methods Mol Biol 2015;1263:243-50.

34. Soding J. Protein homology detection by HMM-HMM comparison. Bioinformatics 2005;21:951-60.

35. Shin WH, Heo L, Lee J, Ko J, Seok C, Lee J. LigDockCSA: proteinligand docking using conformational space annealing. J Comput Chem 2011;32:3226-32.

36. BIOVIA, Dassault Systemes. Discovery studio modeling environment v2021. San Diego: Dassault Systemes; 2016.

37. Manochitra K, Parija SC. In silico prediction and modeling of the entamoeba histolytica proteins: serine-rich entamoeba histolytica protein and $29 \mathrm{kDa}$ cysteine-rich protease. Peer J 2017; 5:3160 
38. Appaiah P, Vasu P. In silico designing of protein rich in large neutral amino acids using bovine $\alpha$ s1 casein for treatment of phenylketonuria. J Proteomics Bioinform 2016;9:287-97.

39. Bagag A, Jault JM, Sidahmed Adrar N, Refregiers M, Giuliani A, Le Naour F. Characterization of hydrophobic peptides in the presence of detergent by photoionization mass spectrometry. PloS One 2013;8:79033.

40. Jones DT. Protein secondary structure prediction based on position-specific scoring matrices. J Mol Biol 1999;292:195-202.

41. Ramachandran S, Kota P, Ding F, Dokholyan NV. Automated minimization of steric clashes in protein structures. Proteins Struct Funct Bioinforma 2011;79:261-70.

42. Wallace AC, Laskowski RA, Thornton JM. Ligplot: a program to generate schematic diagrams of protein-ligand interactions. Protein Eng Des Sel 1995;8:127-34.

43. Jenkinson SG. The use of cefotaxime in the treatment of grampositive pneumonias. Infection 1985;13:14-7.

44. Rolston K, Bolivar R, Fainstein V, Jones P, Elting linda, Bodey GP. Cefotaxime: single agent therapy for infections in cancer patients with adequate granulocyte counts. J Antimicrob Chemother 1985;15:91-6.

45. Lefevre G, Ianotto JC, Tempescul A, Lemoine P, Guillerm G, Berthou C. Infection by H1N1 flu virus revealing T-cell acute lymphoid leukaemia: about two cases. Ann Hematol 2011;90:1111-2.

46. Maiche AG, Teerenhovi L. Empiric treatment of serious infections in patients with cancer: randomised comparison of two combinations. Infection 1991;19:326-9.
47. Leroux S, Roue JM, Gouyon JB, Biran V, Zheng H, Zhao W, et al. A population and developmental pharmacokinetic analysis to evaluate and optimize cefotaxime dosing regimen in neonates and young infants. Antimicrob Agents Chemother 2016;60:6626-34.

48. Wang Y, Xiao J, Suzek TO, Zhang J, Wang J, Bryant SH. PubChem: a public information system for analyzing bioactivities of small molecules. Nucleic Acids Res 2009;37 Suppl 2:623.

49. O’Boyle NM, Banck M, James CA, Morley C, Vandermeersch T, Hutchison GR. Open babel: an open chemical toolbox. J Cheminform 2011;3:1-4.

50. Pettersen EF, Goddard TD, Huang CC, Couch GS, Greenblatt DM, Meng EC, et al. UCSF chimera-a visualization system for exploratory research and analysis. J Comput Chem 2004;25:1605-12.

51. Vimal A, Pal D, Tripathi T, Kumar A. Eucalyptol, sabinene and cinnamaldehyde: potent inhibitors of salmonella target protein l-asparaginase. 3 Biotech 2017;7:1-4.

52. Galande S, Khaursade P, Reddy Shetty P, PB Kavi K, Shetty Prakasham R, Kishor PBK. In silico development of efficient Lasparaginase enzyme for acute lymphoblastic leukaemia therapy. Int J Pharm Sci Res 2018;9:4177-86.

53. Gonzalez Torres I, Perez Rueda E, Evangelista Martinez Z, Zarate Romero A, Moreno Enriquez A, Huerta Saquero A. Identification of L-asparaginases from Streptomyces strains with competitive activity and immunogenic profiles: a bioinformatic approach. Peer J 2020;8:10276. 